\title{
Role of peak current in conversion of patients with ventricular fibrillation
}

\author{
Venkataraman Anantharaman ${ }^{1}$, MBBS, FRCPEd, Paul Weng Wan $^{1}$, MBBS, MMed, Seow Yian Tay $^{2}$, MBBS, FRCS, \\ Peter George Manning ${ }^{3}$, MBBS, FAMS, Swee Han Lim ${ }^{1}$, FRCS, FRCP, Siang Jin Terrance Chua ${ }^{4}$, MMed, MRCP, \\ Tiru Mohan ${ }^{5}$, MB BCh, FRCS, Antony Charles Rabind ${ }^{6}$, MBBS, FRCS, Sudarshan $\underline{\text { Vidya }}^{1}, \mathrm{MSc}, \mathrm{PhD}, \mathrm{Ying}^{\mathrm{HaO}}{ }^{7}, \mathrm{PhD}$
}

INTRODUCTION Peak currents are the final arbiter of defibrillation in patients with ventricular fibrillation (VF). However, biphasic defibrillators continue to use energy in joules for electrical conversion in hopes that their impedance compensation properties will address transthoracic impedance (TTI), which must be overcome when a fixed amount of energy is delivered. However, optimal peak currents for conversion of VF remain unclear. We aimed to determine the role of peak current and optimal peak levels for conversion in collapsed VF patients.

METHODS Adult, non-pregnant patients presenting with non-traumatic VF were included in the study. All defibrillations that occurred were included. Impedance values during defibrillation were used to calculate peak current values. The endpoint was return of spontaneous circulation (ROSC).

RESULTS Of the 197 patients analysed, 105 had ROSC. Characteristics of patients with and without ROSC were comparable. Short duration of collapse $<10$ minutes correlated positively with ROSC. Generally, patients with average or high TTI converted at lower peak currents. $25 \%$ of patients with high TTI converted at $13.3 \pm 2.3 \mathrm{~A}, 22.7 \%$ with average TTI at $18.2 \pm 2.5 \mathrm{~A}$ and $18.6 \%$ with low TTI at $27.0 \pm 4.7 \mathrm{~A}(\mathrm{p}=0.729)$. Highest peak current conversions were at $<15 \mathrm{~A}$ and 15-20 A. Of the 44 patients who achieved first-shock ROSC, $33(75.0 \%)$ received $<20 \mathrm{~A}$ peak current vs. $>20 \mathrm{~A}$ for the remaining $11(25 \%)$ patients $(p=0.002)$.

CONCLUSION For best effect, priming biphasic defibrillators to deliver specific peak currents should be considered.

Keywords: collapse duration, defibrillation energy, electrical conversion, peak current, transthoracic impedance

\section{INTRODUCTION}

Most of the currently available defibrillators use biphasic energy in the form of joules to deliver shocks to patients in either ventricular fibrillation (VF) or pulseless ventricular tachycardia (VT). There are various forms and shapes of biphasic energy, such as biphasic truncated exponential waveform, rectilinear biphasic waveform or pulsed biphasic waveform. Shocks as efficacious as, or superior to, monophasic damped sine wave shocks of the same energy level have been demonstrated with biphasic defibrillators in both animal and human models. ${ }^{(1-4)}$ Higher levels of biphasic energy may be more efficacious in the ranges usually used for monophasic defibrillation (viz. up to $360 \mathrm{~J}$ ). ${ }^{(2)}$ This is well demonstrated in some patients with atrial fibrillation. ${ }^{(5)}$

Successful defibrillation appears to be dependent on depolarisation of most of the myocardium. ${ }^{(6)}$ Energy has to pass through various chest wall layers before reaching electrically sensitive fibrillating myocardial fibres. These layers impose some amount of resistance to the conduction of electrical energy. This is referred to as transthoracic impedance (TTI). The higher the TTI, the less the current that reaches the heart. While a high-amplitude current shock ensures depolarisation of the myocardium, it may cause damage to cardiac tissue. ${ }^{(7)}$ The success of defibrillation depends not primarily on energy levels, but more on peak current flow across the heart ${ }^{(8-12)}$ and other variables such as
$\mathrm{TTI}^{,{ }^{(10)}}$ as well as time elapsed from onset of VF to delivery of first shock. ${ }^{(13,14)}$ Thus, successful cardioversion entails lower TTI and the delivery of an optimal current. Impedance-compensating defibrillators are commonly used, and many clinicians currently believe that differences in TTI values have no major impact on the success of defibrillation. ${ }^{(15,16)}$ However, controversy surrounds the effectiveness of these impedance-compensating processes, and there continues to be a strong belief that TTI significantly affects the ability of modern-day defibrillators to deliver the appropriate level of current to the fibrillating myocardium. ${ }^{(17-19)}$

The level of peak current required to convert VF in humans needs to be defined. Current levels of peak current used are not related in any linear manner to energy delivered. Neither are current energy levels known to cause notable myocardial damage..$^{(8-12)}$ At least three laboratory studies $^{(8,9,20)}$ have demonstrated that higher peak current was related to increased survival, whereas high and high-average currents were associated with post-resuscitation myocardial dysfunction. There is, however, a paucity of such evidence in the clinical environment in humans. Previous laboratory-based studies on induced VF have shown varying levels of peak current for conversion. ${ }^{(20-23)}$ Animal studies suggest a peak current value of $16.4 \pm 2.5 \mathrm{~A}^{(24)}$ for first-shock effect. The aim of this study was to determine the role, if any, of peak current in the conversion of patients who

\footnotetext{
${ }^{1}$ Department of Emergency Medicine, Singapore General Hospital, ${ }^{2}$ Emergency Department, Tan Tock Seng Hospital, ${ }^{3}$ Emergency Medicine Department, National University Hospital, ${ }^{4}$ Department of Cardiology, National Heart Centre Singapore, ${ }^{5}$ Accident and Emergency Department, Changi General Hospital, ${ }^{6} \mathrm{Accident}$ and Emergency Department, Ng Teng Fong General Hospital, ${ }^{7}$ Health Services Research Unit, Singapore General Hospital, Singapore

Correspondence: Prof Venkataraman Anantharaman, Senior Consultant, Department of Emergency Medicine, Singapore General Hospital, Outram Road, Singapore 169608. anantharaman@singhealth.com.sg
} 
had collapsed from VF and the optimal peak levels, if any, for conversion.

\section{METHODS}

This was part of a prospective randomised controlled trial involving four hospitals. The study compared the effectiveness of low biphasic energy and higher biphasic escalating energy in inpatient management of VF. ${ }^{(25)}$ The trial was registered on www. clinicaltrials.gov (NCT00429611).

\section{Study design, interventions and population}

Patients with either VF or pulseless VT were defibrillated with biphasic energy, initially at $150 \mathrm{~J}$ for the low energy group and $200 \mathrm{~J}$ for the higher escalating energy group. If first-shock conversion did not occur, further shocks were given until the patient was no longer in a shockable rhythm. For those in the low energy group, further shocks, if any, were given at $150 \mathrm{~J}$, $150 \mathrm{~J}$ and, if still in VF, increased to $200 \mathrm{~J}$, then to high levels at $300 \mathrm{~J}, 360 \mathrm{~J}$ and further at $360 \mathrm{~J}$ till either conversion or asystole. For those in the higher energy group, further shocks, if any, were given at $300 \mathrm{~J}$ and, if still in VF, more shocks at $360 \mathrm{~J}$ were administered. For all patients, TTI was measured for each shock administered. The primary endpoint was return of spontaneous circulation (ROSC).

All study sites used LIFEPAK $\mathbb{B} 12$ Biphasic Defibrillator (Physio-Control Inc, Redmond, WA, USA) in the manual mode. This was to ensure that defibrillation waveform differences would not be a factor affecting outcomes. If VF was detected, the patient would be included and the randomised defibrillation protocol for that site and week instituted.

Eligible participants were cardiac arrest patients aged $\geq 21$ years with VF. Patients aged $<21$ years, and those with trauma arrest or known to be pregnant were excluded. Patients who had any other shockable rhythms (VT or supraventricular tachycardia) with a pulse and those who were haemodynamically unstable were also excluded from the trial. Cluster block randomisation by week was done prior to admission of the patient. Every week, each study centre's defibrillators were randomly allocated to either of the treatment arms (i.e. higher escalating energy of 200-300-360 J or low energy of 150-150-150 J). The random selection process was done independently by the envelope method.

\section{Determination of peak current delivered ${ }^{(26-28)}$}

1. The TTI of an individual was measured by the delivery of a low-amplitude, high-frequency $(20 \mathrm{kHz})$ current between the two pads prior to the shock being delivered. An impedance value was reflected in the code summary sheet of the LIFEPAK 12 defibrillator. The true shock impedance (Zs) was then calculated using the formula:

$$
\mathrm{Zs}=\frac{(\mathrm{Zvc}-5.10)}{0.88}
$$

where Zvc (voltage compensation impedance) refers to the value reflected in the code summary report.
Table I. Nominal capacitor voltages for selected biphasic energy settings.

\begin{tabular}{lc}
\hline $\begin{array}{l}\text { Selected biphasic } \\
\text { energy setting }(\boldsymbol{J})\end{array}$ & $\begin{array}{c}\text { Nominal capacitor } \\
\text { voltage }(\mathbf{V})\end{array}$ \\
\hline 150 & 1,372 \\
200 & 1,585 \\
300 & 1,941 \\
360 & 2,126 \\
\hline
\end{tabular}

2. The value of the peak current generated prior to the administration of shock was calculated using the following formula: Peak current $=($ Final charge voltage $) /\left(Z S+5^{*}\right)$. This formula presumed that the defibrillator had a nominal internal resistance of 5 ohms.

3. The final charge voltage was determined using the formula: Final charge voltage $=$ Nominal capacitor voltage + Voltage compensation boost

The nominal capacitor voltages used for selected biphasic energy settings are shown in Table I. If TTI was $\leq 60$ ohms, the final charge voltage was presumed to be equal to the nominal capacitor voltage as indicated in Table I. If TTI was 60-200 ohms, a voltage compensation boost had to be factored in to push the current across the chest. The voltage compensation boost was determined as follows:

Voltage compensation boost $=\frac{\{(\mathrm{Zvc} \times 0.1057)-6.27\} \times \mathrm{V}}{100}$

For TTI values > 200 ohms, a value for Zvc as 200 ohms was used. (Note: the maximum final charge voltage is limited to $2,126 \mathrm{~V}$, regardless of energy setting and impedance. Thus, if voltage compensation boost + nominal capacitor voltage is $>2,126 \mathrm{~V}$, then $2,126 \mathrm{~V}$ would still be used as the final value. At the $360 \mathrm{~J}$ energy setting, there is no voltage compensation, and the final charge voltage is always $2,126 \mathrm{~V}$.)

\section{Informed consent and good clinical practice issues}

The trial was approved by the Clinical Trials Coordinating Committee of Ministry of Health Singapore and the respective institutional review boards (IRBs) of the participating institutions. Waiver of informed consent was given for conducting the interventions at the time of cardiac arrest presentation. Within six weeks after the arrest, written approval to use the patient's trial data and information from clinical records was obtained from the patients (for survivors), next-of-kin or legal representative (for non-survivors). If we were unable to establish contact with the patient or next-of-kin, as appropriate, at least three documented attempts at contact (including telephone call records and letters to the last known postal address) were made and kept for potential inspection by the IRBs. Meetings with local ethnic and religious leaders were conducted for feedback and advice on informed consent issues that affected each community group. Public consultation was conducted via a public forum to explain the trial to and obtain immediate feedback from members of the public, followed by announcements about the trial through print/ broadcast media and a period for further feedback from the public. 
Table II. Characteristics of ventricular fibrillation patients for peak current analysis.

\begin{tabular}{|c|c|c|c|c|}
\hline \multirow[t]{2}{*}{ Patient characteristic } & \multicolumn{3}{|c|}{ No. (\%) } & \multirow[t]{2}{*}{ p-value } \\
\hline & Total $(n=197)$ & $\operatorname{ROSC}(n=105)$ & No ROSC $(n=92)$ & \\
\hline Race & & & & 0.178 \\
\hline Chinese & $127(64.5)$ & 65 (61.9) & $62(67.4)$ & \\
\hline Indian & $23(11.7)$ & $13(12.4)$ & $10(10.9)$ & \\
\hline Malay & $28(14.2)$ & $18(17.1)$ & $10(10.9)$ & \\
\hline Unknown & $6(3.0)$ & $5(4.8)$ & $1(1.1)$ & \\
\hline Male gender & $145(73.6)$ & $72(68.6)$ & $73(79.3)$ & 0.121 \\
\hline Mean age \pm SD $(y r)$ & $63.2 \pm 15.5$ & $62.9 \pm 14.3$ & $63.5 \pm 16.8$ & 0.558 \\
\hline \multicolumn{5}{|l|}{ Pre-existing illness } \\
\hline Hypertension & $87(44.2)$ & $55(52.4)$ & $32(34.8)$ & 0.019 \\
\hline Diabetes mellitus & $47(23.9)$ & $30(28.6)$ & $17(18.5)$ & 0.136 \\
\hline Hyperlipidaemia & $76(38.6)$ & $50(47.6)$ & $26(28.3)$ & 0.008 \\
\hline Heart disease & $74(37.6)$ & $38(36.2)$ & $36(39.1)$ & 0.781 \\
\hline
\end{tabular}

ROSC: return of spontaneous circulation; SD: standard deviation

\section{Data collection and analysis}

Data items that were captured included patient demographics, duration of cardiac arrest, TTI prior to each attempt at defibrillation, energy levels (in joules) used, and outcome of each shock administered. For the purpose of peak current sub-analysis, only patients who had either VF or pulseless VT and had documentation of TTI values for shocks administered were included. The primary endpoint was ROSC.

Continuous variables were summarised with mean and standard deviation (SD), and categorical variables with number and percentage. For comparison of demographic characteristics with the primary endpoint (i.e. ROSC), as well as comparisons of different durations of collapse and TTI values, Mann-Whitney $U$ test/ANOVA was used for continuous variables and chi-square/ Fisher's exact test was used for categorical variables. Statistical significance was defined as $p<0.05$. All statistical analyses were performed using R 3.3.1.

\section{RESULTS}

During the study period, 245 patients who sustained cardiac arrest as a result of VF or pulseless VT were recruited for the study (Fig. 1). Eight patients were excluded, as they did not fulfil the inclusion criteria. Two patients were inadvertently recruited twice, while 38 patients did not have records of their TTI values, which were crucial for calculation of peak current. Of the 197 patients who were eligible for analysis, 145 (73.6\%) were male. The mean age of the patients was 63.2 years. The detailed characteristics of the study patients are shown in Table II.

A total of 105 (53.3\%) patients achieved ROSC. There was no significant difference in patient characteristics between patients who achieved ROSC and those who did not, except that a higher proportion of those with ROSC had known prior hypertension or hyperlipidaemia.

\section{Impact of duration of collapse on outcomes (Table III)}

(a) The mean duration of collapse for those who achieved ROSC vs. those who did not was $15.9 \pm 17.3$ minutes vs. $32.3 \pm$

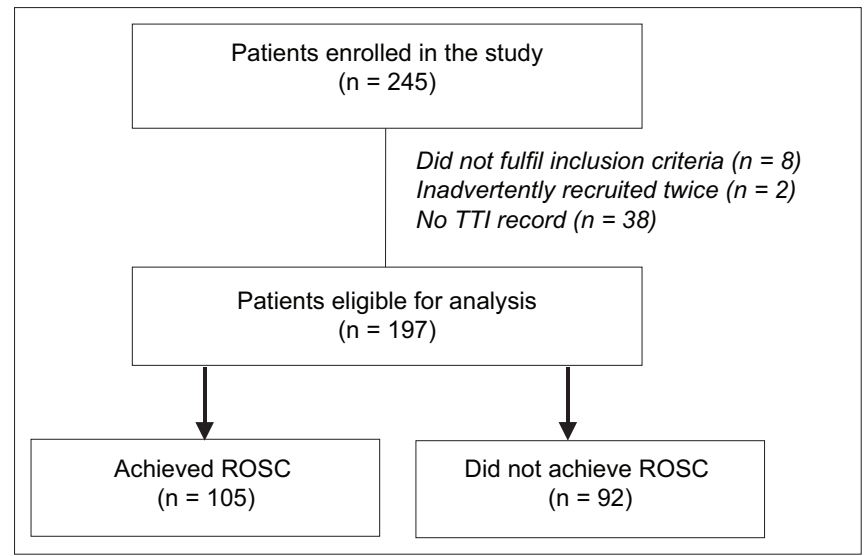

Fig. 1 CONSORT diagram for peak currents in conversion of ventricular fibrillation patients. ROSC: return of spontaneous circulation; TTI: transthoracic impedance

20.1 minutes $(p<0.001)$. For those who achieved ROSC, $55.2 \%$ had duration of collapse $<10$ minutes.

(b) Patients with duration of collapse $<10$ minutes had an overall ROSC rate of $80.6 \%$ vs. $34.1 \%$ for those with duration of collapse $>30$ minutes $(p<0.001)$.

(c) For the 72 patients with short duration of collapse (<10 minutes), 41 (56.9\%) required low energy (i.e. $\leq 200 \mathrm{~J}$ ) for conversion, while only 13 out of 85 patients (15.3\%) with long duration of collapse (> 30 minutes) were converted with low energy $(p=0.082)$.

(d) The impact of duration of collapse was less when higher energy shocks were used.

(e) The first-shock ROSC rate was $41.7 \%$ for patients with short duration of collapse ( $<10$ minutes) vs. $9.4 \%$ for those with long duration of collapse ( $>30$ minutes) $(p=0.075)$. There was no significant difference in $\mathrm{VF}$ recurrence rates between the two groups.

TTI, energy levels and peak currents (Table IV)

More than half of the patients $(110 / 197,55.8 \%)$ had average TTI values (61-90 ohms). In our study cohort, the impedance 
Table III. Collapse duration, relationship to return of spontaneous circulation (ROSC) and peak currents at conversion.

\begin{tabular}{|c|c|c|c|c|c|c|c|c|}
\hline \multirow{2}{*}{$\begin{array}{l}\text { Collapse duration } \\
\text { (min) }\end{array}$} & \multirow{2}{*}{$\begin{array}{c}\text { No. of } \\
\text { patients }\end{array}$} & \multicolumn{4}{|c|}{ ROSC } & \multirow[t]{2}{*}{ No ROSC } & \multirow{2}{*}{$\begin{array}{l}\text { Peak current at } \\
\text { ROSC* (A) }^{*}\end{array}$} & \multirow{2}{*}{$\begin{array}{l}\text { Recurrence } \\
\text { rate of VF }\end{array}$} \\
\hline & & Total & $\leq 200 \mathrm{~J}$ & $360 \mathrm{~J}$ & 1st shock & & & \\
\hline $0-10$ & 72 & $58(80.6)$ & 41 (56.9) & $13(18.1)$ & $30(41.7)$ & $14(19.4)$ & $19.5 \pm 6.5$ & $14(24.1)$ \\
\hline $10-20$ & 19 & $8(42.1)$ & 7 (36.8) & $1(5.3)$ & $4(21.1)$ & $11(57.9)$ & $20.1 \pm 3.5$ & $3(37.5)$ \\
\hline $20-30$ & 21 & $10(47.6)$ & $5(23.8)$ & $4(19.0)$ & $2(9.5)$ & $11(52.4)$ & $28.2 \pm 14.7$ & $2(20.0)$ \\
\hline$>30$ & 85 & $29(34.1)$ & 13 (15.3) & $9(10.6)$ & $8(9.4)$ & $56(65.9)$ & $22.4 \pm 8.1$ & $9(31.0)$ \\
\hline$p$-value & & $<0.001$ & 0.082 & & 0.075 & & 0.014 & 0.754 \\
\hline
\end{tabular}

Data presented as no. (\%) unless otherwise stated. *Data presented as mean \pm standard deviation. VF: ventricular fibrillation

Table IV. Transthoracic impedance, energy levels and peak currents for conversion.

\begin{tabular}{|c|c|c|c|c|}
\hline \multirow[t]{2}{*}{ Parameter } & \multicolumn{3}{|c|}{ Transthoracic impedance (ohms) } & \multirow[t]{2}{*}{ p-value } \\
\hline & Low $(\leq 60)(n=43)$ & Average $(61-90)(n=110)$ & High $(>90)(n=44)$ & \\
\hline Achieved ROSC & $20(46.5)$ & $58(52.7)$ & $27(61.4)$ & 0.729 \\
\hline At first shock & $8(18.6)$ & $25(22.7)$ & $11(25.0)$ & \\
\hline At low energy $(\leq 200 \mathrm{~J})$ & $11(25.6)$ & $40(36.4)$ & $15(34.1)$ & 0.138 \\
\hline At high energy $(360 \mathrm{~J})$ & $4(9.3)$ & $15(13.6)$ & $8(18.2)$ & \\
\hline Peak current at first shock* (A) & $27.0 \pm 4.7$ & $18.2 \pm 2.5$ & $13.3 \pm 2.3$ & $<0.001$ \\
\hline Peak current at ROSC* (A) & $30.6 \pm 9.9$ & $20.3 \pm 5.5$ & $15.3 \pm 3.1$ & $<0.001$ \\
\hline$<15$ & $0(0.0)$ & $10(9.1)$ & $12(27.3)$ & $<0.001$ \\
\hline $15-20$ & $0(0.0)$ & $23(20.9)$ & $14(31.8)$ & \\
\hline $20-25$ & $7(16.3)$ & $13(11.8)$ & $1(2.3)$ & \\
\hline$>25$ & $13(30.2)$ & $12(10.9)$ & $0(0.0)$ & \\
\hline
\end{tabular}

Data presented as no. (\%) unless otherwise stated. *Data presented as mean \pm standard deviation. ROSC: return of spontaneous circulation

values ranged from 32 ohms to 154 ohms. The mean impedance value for those who achieved ROSC vs. those who did not was $78 \pm 21.4$ ohms vs. $73.8 \pm 20.4$ ohms $(95 \%$ confidence interval -1.6 to $4.2 ; \mathrm{p}=0.321)$. Even among patients who achieved first-shock conversion, there was no significant difference in either the initial TTI values or energy levels used. However, among those who required multiple shocks, $12(11.4 \%)$ received $<15 \mathrm{~A}, 21(20.0 \%)$ received $15-20 \mathrm{~A}, 7(6.7 \%)$ received $20-25 \mathrm{~A}$, and $4(3.8 \%)$ received $>25 \mathrm{~A}(\mathrm{p}=0.002)$.

Among patients with low TTI values, the mean peak current generated at first shock was $27.0 \pm 4.7 \mathrm{~A}$, with a first-shock conversion rate of $18.6 \%$. For those with average TTI values, the mean peak current at first shock was $18.2 \pm 2.5 \mathrm{~A}$, with a firstshock ROSC rate of $22.7 \%$. For patients with high TTI values, the mean peak current generated was only $13.3 \pm 2.3 \mathrm{~A}$; this group achieved a first-shock ROSC rate of $25.0 \%$.

For patients with low TTI values, the highest rate of conversions to ROSC occurred with peak currents $>25 \mathrm{~A}$. None of these patients converted with peak currents $<20 \mathrm{~A}$. For patients with average and high TTI values, the highest conversion rate was achieved with peak currents of 15-20 A.

The peak current generated among patients who achieved ROSC was 8.96-37.39 A. Only two patients with peak current $<10$ A achieved ROSC, and only eight with peak current > $30 \mathrm{~A}$ had ROSC. The remaining 95 patients achieved ROSC with peak currents of 10-30 A.

\section{DISCUSSION}

About thirty years ago, it was suggested that current-based defibrillation, rather than energy-based defibrillation, would preclude TTI as a significant determinant of shock success and deliver significantly less current and energy to patients with equivalent success. ${ }^{(20)}$ With the advent of built-in impedance compensation in biphasic defibrillators, variances in TTI in different patients were thought to be relatively unimportant for delivering the level of electrical energy required by patients for electrical conversion. The belief that TTI does not affect defibrillation, resuscitation or survival in cardiac arrest patients treated with a nonescalating biphasic waveform defibrillator was extremely strong. In spite of this, most of the defibrillators in use today continue to be energy-based, i.e. users select the energy levels (in joules) they wish to employ to defibrillate the patient but do not select the amount of current (in amperes) to deliver to the heart.

In performing electrical defibrillation for patients in $\mathrm{VF}$, one looks for first-shock success. Yet, many patients require more than one shock, and in some instances, higher energy shocks must be applied before electrical conversion occurs. ${ }^{(25)}$ In these patients, one may question whether the impedance-compensating mechanisms of the defibrillators have faltered, thus requiring the use of higher energy levels. ${ }^{(29)}$ A 2003 study comparing six commercially available biphasic defibrillators ${ }^{(17)}$ that used different electrical waveforms noted that, while efficacy with these waveforms was fairly uniform at low impedance values, there were significant variations at higher impedance values. 
The study concluded that despite impedance-compensation schemes in biphasic defibrillators, impedance has an impact on defibrillation efficacy. ${ }^{(17)}$

In the present study, we could not establish that patients with low TTI values were easier to defibrillate. On the contrary, we found that the success rate in such patients was lower than for those with average or high TTI values, although not significantly so. Even then, the higher currents generated for such patients may not have been optimal, even with the use of low energy shocks. The main factors that positively contributed to higher defibrillation success in this study appear to be a shorter duration of collapse and patients with higher TTI receiving lower peak currents.

Initial studies on peak currents required for defibrillation in small and large animals revealed a desired range of $0.5-2.0 \mathrm{~A} / \mathrm{kg}$ body weight. ${ }^{(30)} \mathrm{A}$ study on peak currents required during open heart surgery ${ }^{(31)}$ noted that the lowest range of currents to successfully defibrillate the heart was 8-34 A. Peak currents measured during defibrillation of VF patients in a coronary care unit averaged $0.35 \mathrm{~A} / \mathrm{kg}$ body weight. ${ }^{(32)} \mathrm{A}$ swine model for $\mathrm{VF}$ cardiac arrest suggested 12.8-17.0 A. ${ }^{(24)}$ However, laboratorybased human studies propose an optimal range of current of 18-54 A for human defibrillation. ${ }^{(20-23)}$

In the present study, the peak currents that were associated with the best rates of ROSC for patients with average and high TTI were 15-20 A, although some patients with these TTI values converted at $<15$ A of peak current. Patients with low TTI could not have received lower peak currents, because even low energies of 150-200 J resulted in the delivery of at least $20 \mathrm{~A}$ of peak current. Some of the highest peak currents in this study were used in patients with low TTI. This is of concern, as the very high peak currents generated may result in the formation of membrane microlesions at the regions of high current density, ${ }^{(33)}$ which would increase the chance of post-shock myocardial dysfunction. This suggests that high-current shocks should be avoided, especially in low TTI patients, to reduce the chances of myocardial damage.

Therefore, optimal peak currents for electrical conversion of patients with VF appear to be in the range of 10-20 A. Since patients with short duration of cardiac arrest require a slightly lower peak current for conversion, it would appear that one should aim for low currents when initially defibrillating patients with VF. The question is whether a first attempt at defibrillation should aim to deliver a relatively low peak current of 10-15 A. Upon failure to convert, one may then consider escalating the peak current delivered to 15-20 A. For patients who do not convert at these relatively low current levels, higher currents may be required.

If our conviction that peak currents rather than energy in joules are the final arbiter of the ability to convert VF holds true, then defibrillators should be primed to deliver specific peak currents, rather than energy levels in joules, for best effect. Further research in the use of reliable, specific current delivery mechanisms is required to better appreciate the value of peak current in defibrillation of patients with VF.

The present study is not without limitations. We determined the peak currents achieved through a calculated formula rather than by direct ampere measurement. Thus, any approximations used in this calculation could have affected the values demonstrated. Future studies should measure the current generated with appropriate direct ammeter readings.

\section{ACKNOWLEDGEMENTS}

The study was sponsored in part by the National Medical Research Council, Medtronic Emergency Response Systems (USA) and Singapore Clinical Research Institute. The study sponsors had no role in the study design, data collection, data analysis, data interpretation and writing of the report. The corresponding author had full access to all the data and takes final responsibility for the decision to submit this paper for publication. The co-authors do not have any conflicts of interest with the study sponsors.

\section{REFERENCES}

1. Koster RW, Dorian P, Chapman FW, Schmitt PW, O'Grady SG, Walker RG. A randomized trial comparing monophasic and biphasic waveform shocks for external cardioversion of atrial fibrillation. Am Heart J 2004; 147:e20.

2. Niemann JT, Burian D, Garner D, Lewis RJ. Monophasic versus biphasic transthoracic countershock after prolonged ventricular fibrillation in a swine model. J Am Coll Cardiol 2000; 36:932-8.

3. Bardy GH, Marchlinski FE, Sharma AD, et al. Multicenter comparison of truncated biphasic shocks and standard damped sine wave monophasic shocks for transthoracic ventricular defibrillation. Transthoracic Investigators. Circulation 1996; 94:2507-14.

4. Tang W, Weil MH, Sun S, et al. A comparison of biphasic and monophasic waveform defibrillation after prolonged ventricular fibrillation. Chest 2001; 120:948-54.

5. Jain VC, Wheelan K. Successful cardioversion of atrial fibrillation using 360-Joules biphasic shock. Am J Cardiol 2002; 90:331-2.

6. Chen CJ, Guo GB. External cardioversion in patients with persistent atrial fibrillation: a reappraisal of the effects of electrode pad position and transthoracic impedance on cardioversion success. Jpn Heart J 2003; 44:921-32.

7. Babbs CF, Tacker WA, VanVleet JF, Bourland JD, Geddes LA. Therapeutic indices for transchest defibrillator shocks: effective, damaging, and lethal electrical doses. Am Heart J 1980; 99:734-8.

8. Higgins SL, O'Grady SG, Banville I, et al. Efficacy of lower-energy biphasic shocks for transthoracic defibrillation: a follow-up clinical study. Prehosp Emerg Care 2004; 8:262-7.

9. Tang W, Weil MH, Sun S, et al. The effects of biphasic waveform design on post-resuscitation myocardial function. J Am Coll Cardiol 2004; 43:1228-35.

10. Budde T, Vukmirovic NB, Soriano-Romero JM, et al. Bidirectional transvenous/ subcutaneous defibrillation of ventricular fibrillation in dogs: success rates, energy requirements, currents, voltages and impedance. Eur Heart J 1988; 9:92-101.

11. Kontos MC, Ellenbogen KA, Wood MA, et al. Factors associated with elevated impedance with a nonthoracotomy defibrillation lead system. Am J Cardiol 1997; 79:48-52.

12. Dorian P, Wang MJ. Defibrillation current and impedance are determinants of defibrillation energy requirements. Pacing Clin Electrophysiol 1988; 11(11 Pt 2):1996-2001.

13. Newman M. Early defibrillation. Making waves across America. JEMS 1997; 22:S4-8.

14. Ladwig KH, Schoefinius A, Danner R, et al. Effects of early defibrillation by ambulance personnel on short- and long-term outcome of cardiac survival: the Munich experiment. Chest 1997; 112:1584-91.

15. Dalzell GW, Anderson J, Adgey AA. Factors determining success and energy requirements for cardioversion of atrial fibrillation: revised version. Q J Med $1991 ; 78: 85-95$.

16. White RD, Blackwell TH, Russell JK, Snyder DE, Jorgenson DB. Transthoracic impedance does not affect defibrillation, resuscitation or survival in patients with out-of-hospital cardiac arrest treated with a non-escalating biphasic waveform defibrillator. Resuscitation 2005; 64:63-9.

17. Walker RG, Melnick SB, Chapman FW, et al. Comparison of six clinically used external defibrillators in swine. Resuscitation 2003; 57:73-83.

18. Jones JL, Snyder DE, Morgan C. Predictions from misleading pig model are potentially harmful to humans. Resuscitation 2003; 59:365-7.

19. Kerber RE, Kouba C, Martins J, et al. Advance prediction of transthoracic impedance in human defibrillation and cardioversion: importance of impedance in determining the success of low-energy shocks. Circulation 1984; 70:303-8.

20. Lerman BB, DiMarco JP, Haines DE. Current-based versus energy-based 
ventricular defibrillation: a prospective study. J Am Coll Cardiol 1988; 12:1259-64.

21. Kerber RE, Kieso RA, Kienzle MG, et al. Current-based transthoracic defibrillation. Am J Cardiol 1996; 78:1113-8.

22. Kerber RE, Martins JB, Kienzle MG, et al. Energy, current, and success in defibrillation and cardioversion: clinical studies using an automated impedancebased method of energy adjustment. Circulation 1988; 77:1038-46.

23. Kerber RE, Kienzle MG, Olshansky B, et al. Ventricular tachycardia rate and morphology determine energy and current requirements for transthoracic cardioversion. Circulation 1992; 85:158-63.

24. Chen B, Yu T, Ristagno G, Quan W, Li Y. Average current is better than peak current as therapeutic dosage for biphasic waveforms in a ventricular fibrillation pig model of cardiac arrest. Resuscitation 2014; 85:1399-404.

25. Anantharaman V, Tay SY, Manning PG, et al. A multicenter prospective randomized study comparing the efficacy of escalating higher biphasic versus low biphasic energy defibrillations in patients presenting with cardiac arrest in the in-hospital environment. Open Access Emerg Med 2017; 9:9-17.

26. Heavens JP, Cleland, MJ, Maloney JP, Rowe BH. Effects of transthoracic impedance and peak current flow on defibrillation success in a prehospital setting. Ann Emerg Med 1998; 32:191-9.

27. Jones VC, Charbonnier FM, Long P. Determining transthoracic impedance, delivered energy, and peak current during defibrillation episodes. Med Instrum $1981 ; 15: 380-2$

28. Lerman BB, Deale OC. Relation between transcardiac and transthoracic current during defibrillation in humans. Circ Res 1990; 67:1420-6.

29. US Food and Drug Administration. Energy Levels in External Biphasic Defibrillators: Initial Communication. Medical Device Safety Alert Notice dated November 5, 2009. Available at: http://www.fda.gov/MedicalDevices/Safety/ AlertsandNotices/ucm189259.htm. Accessed November 7, 2011.

30. Geddes LA, Tacker WA, Rosborough JP, Moore AG, Cabler PS. Electrical dose for ventricular defibrillation of large and small animals using precordial electrodes. J Clin Invest 1974; 53:310-9.

31. Kerber RE, Carter J, Klein S, Grayzel J, Kennedy J. Open chest defibrillation during cardiac surgery: energy and current requirement. Am J Cardiol 1980; 46:393-6.

32. Patton JN, Pantridge JF. Current required for ventricular defibrillation. Br Med J 1979; 1: 513-4

33. Jones JL, Jones RE, Balasky G, Microlesion formation in myocardial cells by high-intensity electric field stimulation. Am J Physiol 1987; 253(2 Pt 2):H480-6. 